\title{
A Pilot Study to Assess Urban, Fire-Based Paramedic Accuracy in Identification of Anatomical Landmarks Necessary for Cricothyrotomy and Needle Chest Decompression Using Live Patient Models
}

\author{
Romeo R. Fairley; ${ }^{1}$ (1) Sophia Ahmed; ${ }^{1}$ Steven G. Schauer; ${ }^{1,2,3,4}$ (i) David A. Wampler; ${ }^{5}$ (i) Kaori Tanaka; ${ }^{1}$ \\ Bryan Everitt; ${ }^{1}$ Mark K. Sparkman; ${ }^{1}$ Ramon Casanova; ${ }^{6}$ Justin Sifuentes; ${ }^{6}$ Christopher J. Winckler ${ }^{1,5}$
}

1. University of Texas Health Science Center San Antonio, Department of Emergency Medicine, San Antonio, Texas USA

2. United States Army Institute of Surgical Research, San Antonio, Texas USA

3. Brooke Army Medical Center, Fort Sam Houston, Texas USA

4. Uniformed Services University of the Health Sciences, Bethesda, Maryland USA

5. University of Texas Health Science Center San Antonio, Department of Emergency Health Sciences, San Antonio, Texas USA

6. San Antonio Fire Department, San Antonio, Texas USA

Correspondence:

Romeo R. Fairley

University of Texas Health Science Center San Antonio

Department of Emergency Medicine 7703 Floyd Curl Drive, MC 7736

San Antonio, Texas 78229 USA

E-mail: FairleyR@UTHSCSA.edu

Conflicts of interest/funding: This study was supported by a Defense Health Program grant (DP_67.2_17_I_17_J9_1635). No conflicts of interest for any authors to declare.

Keywords: airway; cricothyrotomy; needle chest decompression; paramedic education

\section{Abbreviations: \\ EMS: Emergency Medical Services \\ EMT: emergency medical technicians \\ ICS: intercostal space \\ MTO: medical training officer \\ NDC: needle decompression \\ SAFD: San Antonio Fire Department}

Received: November 5, 2020

Revised: January 6, 2021

Accepted: January 15, 2021

\section{Abstract}

Background: Cricothyrotomy and chest needle decompression (NDC) have a high failure and complication rate. This article sought to determine whether paramedics can correctly identify the anatomical landmarks for cricothyrotomy and chest NDC.

Methods: A prospective study using human models was performed. Paramedics were partnered and requested to identify the location for cricothyrotomy and chest NDC (both mid-clavicular and anterior axillary sites) on each other. A board-certified or board-eligible emergency medicine physician timed the process and confirmed location accuracy. All data were collected de-identified. Descriptive analysis was performed on continuous data; chi-square was used for categorical data.

Results: A total of 69 participants were recruited, with one excluded for incomplete data. The paramedics had a range of six to 38 (median 14) years of experience. There were 28 medical training officers (MTOs) and 41 field paramedics. Cricothyroidotomy location was correctly identified in 56 of 68 participants with a time to identification range of 2.0 to 38.2 (median 8.6) seconds. Chest NDC (mid-clavicular) location was correctly identified in 54 of 68 participants with a time to identification range of 3.4 to 25.0 (median 9.5) seconds. Chest NDC (anterior axillary) location was correctly identified in 43 of 68 participants with a time to identification range of 1.9 to 37.9 (median 9.6) seconds. Chi-square (2-tail) showed no difference between MTO and field paramedic in cricothyroidotomy site $(\mathrm{P}=.62)$, mid-clavicular chest NDC site $(\mathrm{P}=.21)$, or anterior axillary chest NDC site $(\mathrm{P}=.11)$. There was no difference in time to identification for any procedure between MTO and field paramedic.

Conclusion: Both MTOs and field paramedics were quick in identifying correct placement of cricothyroidotomy and chest NDC location sites. While time to identification was clinically acceptable, there was also a significant proportion that did not identify the correct landmarks.

Fairley RR, Ahmed S, Schauer SG, Wampler DA, Tanaka K, Everitt B, Sparkman MK, Casanova R, Sifuentes J, Winckler CJ. A pilot study to assess urban, fire-based paramedic accuracy in identification of anatomical landmarks necessary for cricothyrotomy and needle chest decompression using live patient models. Prehosp Disaster Med. 2021;36(4):408-411.

\section{Introduction}

Cricothyrotomy and needle decompression (NDC) are important, life-saving procedures for which Emergency Medical Services (EMS) personnel need to be trained and well-versed for prehospital patient management. ${ }^{1,2}$

\section{doi:10.1017/S1049023X21000340}

(C) The Author(s), 2021. Published by Cambridge University Press on behalf of World Association for Disaster and Emergency Medicine. This is an Open Access article, distributed under the terms of the Creative Commons Attribution licence (http://creativecommons.org/licenses/by/4.0/), which permits unrestricted re-use, distribution, and reproduction in any medium, provided the original work is properly cited. 
When other airway interventions are not possible, cricothyrotomy is the last resort airway in patients that are critically ill or have wide-scale injuries involving the upper airway anatomy. Consequently, prehospital cricothyrotomy is rare and success rates vary widely by procedure type; needle cricothyrotomy ranges from $25 \%-77 \%$ and surgical cricothyrotomy ranges from $62 \%-100 \%$ when accounting for both EMS personnel and emergency medicine physicians. ${ }^{3}$ In a military-based study, Mabry noted that two-thirds of patients undergoing prehospital cricothyrotomy died, and nearly one-fourth of those attempts were unable to cannulate the trachea. ${ }^{4}$

In patients with a tension pneumothorax undergoing NDC, incorrect catheter placement and failure to cannulate the chest cavity are the most common complications. ${ }^{5}$ Procedural failure rates among EMS and emergency medicine physicians range from $22 \%-55 \%$ based on different studies. ${ }^{1,6,7}$ These studies do not evaluate for anatomical site accuracy. Variation in success is attributable to many factors including specific technique, personnel training and prior experience with the procedure, patient body habitus, and overall patient trauma.

Rapid and accurate identification of key anatomical landmarks by EMS personnel is essential for successful cricothyrotomy and NDC. There are a paucity of data specifically assessing paramedics' ability to correctly identify anatomical landmarks needed for both cricothyrotomy and NDC.

This article sought to determine whether paramedics at a large, urban fire department could correctly identify the anatomical landmarks for cricothyrotomy and NDC. The primary outcome was the ability to identify the correct anatomical landmark for the procedures. Secondarily, time to identification was assessed. Both outcomes compared medic training officers (MTOs) with field paramedics. The primary hypothesis was that MTOs would be more accurate compared to field paramedics due to their experience level.

\section{Methods}

The study is a prospective observational study, utilizing the Strengthening the Reporting of Observational Studies in Epidemiology (STROBE) statement guidelines. ${ }^{8}$ Human models were voluntarily recruited from the San Antonio Fire Department (SAFD; San Antonio, Texas USA). The SAFD serves a response area of over 450 square miles with a population of 1.5 million and responds to 160,000 medical calls for service every year. The study was performed at the University of Texas Health - San Antonio, Emergency Health Sciences Office of Medical Director (OMD; San Antonio, Texas USA), which provides the participants' continuing education training. The University of Texas Health - San Antonio institutional review board deemed the project (protocol number: HSC20180856N) to not be regulated research as defined by DHHS regulations at $45 \mathrm{CFR} 46$ and FDA regulations at 21 CFR 56. Written consent of participants was obtained.

\section{Selection of Participants}

Paramedics from SAFD were recruited to participate. There are two groups of paramedics that work on the ambulances. The MTOs have applied and been accepted to train incoming paramedics; they practice teaching daily and often participate in extra training. Field paramedics perform the regular duties as assigned to paramedics at SAFD. Participants were given a standardized demographics information form to complete.

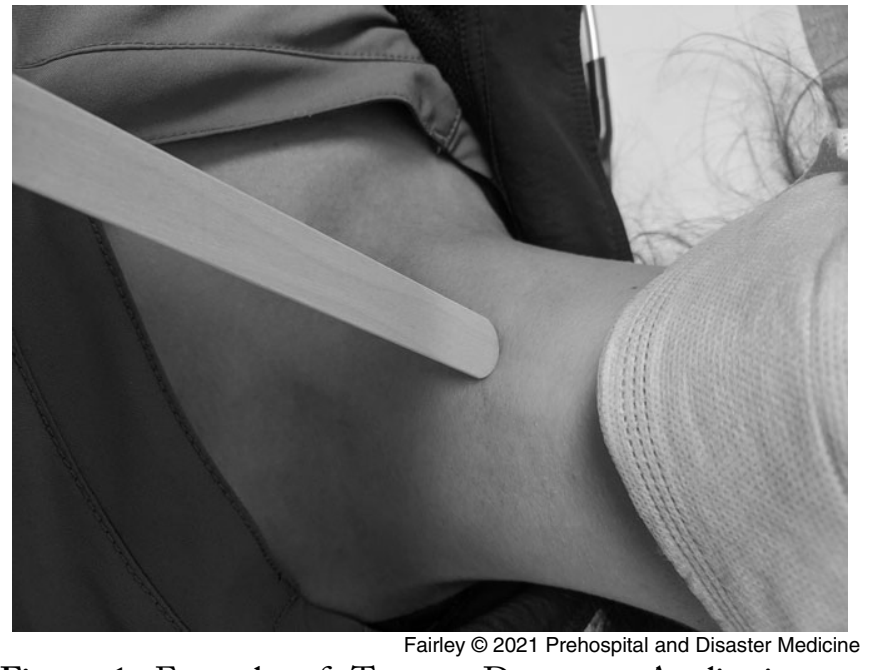

Figure 1. Example of Tongue Depressor Application to Identify the Cricothyroid Membrane.

Lack of existing data regarding the accurate identification of cricothyrotomy or chest NDC landmarks precluded sample size estimation. Based on clinical experience, an estimated 30 participants would be needed to complete the study.

\section{Patient and Public Involvement}

This research was done without patient involvement. Patients were not invited to comment on the study design and were not consulted to develop patient-relevant outcomes or interpret the results. Patients were not invited to contribute to the writing or editing of this document for readability or accuracy.

\section{Method of Measurement}

Participants were split into groups of two. To prevent bias, the pair of medics were then taken to a private area where one member was randomly selected to identify the correct anatomical location for surgical cricothyrotomy using a tongue depressor on his/her partner (Figure 1). The participants then switched roles and repeated the process.

A study team member recorded the time from when the participant first touched the model to the time they identified the anatomical location for the procedure. A board-certified or board-eligible emergency medicine physician that is a member of the study team then confirmed whether the tongue depressor was in the correct location. Next, the placement of the tongue depressor was photographed for secondary verification by another study team member to ensure data collection veracity. No identifying features were included in the photographs (eg, facial features, tattoos, or name tapes). If the two study team members did not agree upon whether the anatomic location was correct, then the opinion from an additional physician was solicited.

In the next phase of study, the medic removed his/her shirt with females specified to leave their sports bra on. A female study team member was present when a female was the model. The medic performing the identification was instructed to identify both correct locations for chest NDC (second intercostal space [ICS] mid-clavicular line and fourth ICS anterior axillary line). Afterwards, the same data collection and photography procedures as outlined above were repeated. 


\begin{tabular}{|l|c|c|c|}
\hline & Minimum & Maximum & Average \\
\hline Age (years) & 27 & 58 & 40 \\
\hline Years of Medic Experience & 6 & 38 & 14 \\
\hline $\begin{array}{l}\text { Number of Surgical Airways } \\
\text { Performed }\end{array}$ & 0 & 50 & 3 \\
\hline $\begin{array}{l}\text { Number of Chest NDCs } \\
\text { Performed }\end{array}$ & 1 & 50 & 10 \\
\hline
\end{tabular}

Table 1. Participant Demographic Information

Abbreviation: NDC, needle decompression.

The medics received feedback on the accuracy of anatomical locations they selected to ensure the study provided an educational benefit.

All data were aggregated and de-identified to obtain further anonymity. All data collection forms were entered into an Excel Database (version 10; Microsoft Corp.; Redmond, Washington USA). All data for analysis was exported into JMP Statistical Discovery from SAS (version 13; SAS Institute; Cary, North Carolina USA). Participant characteristics were compared based upon the technique sequence to which they were assigned by randomization. Categorical variables are reported as numbers with percentages, ordinal variables as medians with interquartile ranges, and continuous variables as means with $95 \%$ confidence intervals.

\section{Results}

A total of 69 participants were recruited, with one excluded for incomplete data. The paramedics had a range of six to 38 (median 14) years of experience (Table 1). There were 28 MTOs and 41 field paramedics. Sixty-six of the 68 participants were male.

Education level was variable, with 64 participants having completed a minimum of some college. Five participants attended graduate school.

Sixty-two of 69 participants reported they had never performed a cricothyrotomy on a patient. Thirteen of 69 participants reported they had never performed chest NDC on a patient. Questions on procedures performed included those on simulations and realworld patients.

Cricothyroidotomy location was correctly identified in 53 of 68 (77.9\%) participants with a time to identification range of 2.0 to 38.2 (median 8.6) seconds. Chest NDC (mid-clavicular) location was correctly identified in 54 of 68 (79.4\%) participants with a time to identification range of 3.4 to 25.0 (median 9.5) seconds. Chest NDC (anterior axillary) location was correctly identified in 43 of $68(63.2 \%)$ participants with a time to identification range of 1.9 to 37.9 (median 9.6) seconds (Table 2).

Chi-squared (2-tail) analysis showed no difference in correct site identification between MTO and field paramedic in cricothyroidotomy site $(\mathrm{P}=.62)$, mid-clavicular chest NDC site $(\mathrm{P}=.21)$, or anterior axillary chest NDC site $(\mathrm{P}=.11)$. There was no difference in time to identification for any procedure between MTO and field paramedic (Table 3 and Table 4). In addition, t-test analysis showed there was no difference in success rates for any procedure when years of experience was compared.

\section{Discussion}

The overall failure rate for the procedures ranged from $22.1 \%$ to $36.8 \%$. This is well within the failure rates previously reported in literature, though this still represents a large portion of potentially missed therapeutic opportunities. Performing both cricothyrotomy

\begin{tabular}{|l|c|c|c|c|}
\hline & $\begin{array}{c}\text { Minimum } \\
\text { (seconds) }\end{array}$ & $\begin{array}{l}\text { Maximum } \\
\text { (seconds) }\end{array}$ & $\begin{array}{c}\text { Average } \\
\text { (seconds) }\end{array}$ & $\begin{array}{c}\text { Accurate } \\
\text { Identification }\end{array}$ \\
\hline $\begin{array}{l}\text { Cricothyroid } \\
\text { Membrane } \\
\text { Identification }\end{array}$ & 2.00 & 38.27 & 8.63 & $77.9 \%$ \\
\hline $\begin{array}{l}\text { Chest NDC } \\
\text { in 2 }\end{array}$ & 3.41 & 25.04 & 9.57 & $78.3 \%$ \\
\hline $\begin{array}{l}\text { Chest NDC } \\
\text { in 4 }\end{array}$ & 1.93 & 37.93 & 9.62 & $63.2 \%$ \\
\hline
\end{tabular}

Fairley $\odot 2021$ Prehospital and Disaster Medicine

Table 2. Time to Identification and Accuracy of Surgical Airway and Chest NDC Sites by Combined MTOs and Field Paramedics

Abbreviations: ICS, intercostal space; MTO, medic training officer; NDC, needle decompression.

\begin{tabular}{|l|c|c|c|c|}
\hline & $\begin{array}{c}\text { Minimum } \\
\text { (seconds) }\end{array}$ & $\begin{array}{l}\text { Maximum } \\
\text { (seconds) }\end{array}$ & $\begin{array}{c}\text { Average } \\
\text { (seconds) }\end{array}$ & $\begin{array}{c}\text { Accurate } \\
\text { Identification }\end{array}$ \\
\hline $\begin{array}{l}\text { Cricothyroid } \\
\text { Membrane } \\
\text { Identification }\end{array}$ & 2.37 & 32.05 & 10.69 & $75.0 \%$ \\
\hline $\begin{array}{l}\text { Chest NDC } \\
\text { in 2 }\end{array}$ & 3.46 & 23.79 & 11.28 & $85.7 \%$ \\
\hline $\begin{array}{l}\text { Chest NDC } \\
\text { in 4 }\end{array}$ & 2.98 ICS & 21.42 & 11.49 & $51.9 \%$ \\
\hline
\end{tabular}

Fairley @ 2021 Prehospital and Disaster Medicine

Table 3. Time to Identification and Accuracy of Surgical Airway and Chest NDC Sites by MTOs

Abbreviations: ICS, intercostal space; MTO, medic training officer; NDC, needle decompression.

\begin{tabular}{|l|c|c|c|c|}
\hline & $\begin{array}{c}\text { Minimum } \\
\text { (seconds) }\end{array}$ & $\begin{array}{c}\text { Maximum } \\
\text { (seconds) }\end{array}$ & $\begin{array}{c}\text { Average } \\
\text { (seconds) }\end{array}$ & $\begin{array}{c}\text { Accurate } \\
\text { Identification }\end{array}$ \\
\hline $\begin{array}{l}\text { Cricothyroid } \\
\text { Membrane } \\
\text { Identification }\end{array}$ & 2.00 & 38.27 & 10.71 & $80.0 \%$ \\
\hline $\begin{array}{l}\text { Chest NDC in } \\
2^{\text {nd }} \text { ICS }\end{array}$ & 3.41 & 25.04 & 10.32 & $73.2 \%$ \\
\hline $\begin{array}{l}\text { Chest NDC in } \\
4^{\text {th }} \text { ICS }\end{array}$ & 1.93 & 37.93 & 10.51 & $70.7 \%$ \\
\hline
\end{tabular}

Fairley $\odot 2021$ Prehospital and Disaster Medicine

Table 4. Time to Identification and Accuracy of Surgical Airway and Chest NDC Sites by Field Paramedics

Abbreviations: ICS, intercostal space; NDC, needle decompression.

and chest NDC requires correct identification of anatomical landmarks followed by procedural execution.

These data show that MTOs were not more accurate or quicker in identification of anatomical location for any of the procedures. There are multiple potential reasons for this. This fire department utilizes a cadaver anatomy laboratory in which recurrent training on procedures is performed for both MTOs and field paramedics. Anecdotally, participants acknowledged the usefulness and their perceived benefits of the cadaver training.

The data from this study can be used to further understand the performance capability and training standards for prehospital EMS personnel. Both MTOs and field paramedics were accurate and quick in identifying correct placement of cricothyroidotomy and chest NDC location sites. Recent procedural reviews have advocated for use of the anterior axillary NDC site due to decreased body mass compared to the mid-clavicular site in obese patients. ${ }^{6}$ 
This study was not designed to show a difference in site identification accuracy; however, a reduced precision in the anterior axillary site identification was evident.

For procedures that are rarely performed, including cricothyrotomy and chest NDC, opportunities to review procedural skills are essential for successful patient care. This study was not designed or powered to evaluate for the specific reasons behind failure to accurately locate the anatomical site. Further studies are warranted to evaluate the maximum benefit of various training techniques. A device to assist in anatomical location identification could be beneficial.

\section{Limitations}

This study has several limitations. First, focus on two fire stations that have a medical special operations unit in an urban fire department resulted in a convenience sample of 69 majority male participants. However, this may limit the extrapolation of these results to EMS personnel at-large and these data likely underrepresents the misidentification rates. Moreover, only paramedic level and above were included - in rural areas emergency medical technicians (EMTs; formerly EMT-Basic) and advanced EMTs (formerly EMT-Intermediate) may have to perform these procedures or assist in their performance which could carry higher complication rates. In addition, some research has shown axillary ICS four and five to be safe and effective for thoracostomy; however, this study only evaluated the fourth ICS identification. A future study can expand upon the findings and include EMS personnel both in rural areas and other urban cities that have different training regimens. Second, since volunteers were used instead of standardized manikins, each participant had a different body habitus resulting in a variety of both neck circumference size and overall body mass. While this better represents the variety in the population for which they must perform this procedure, it may have also skewed the results. Additionally, the study population may underrepresent potential future patients that are extremely obese.

\section{Conclusion}

Both MTOs and field paramedics were quick in identifying correct placement of cricothyroidotomy and chest NDC location sites. While time to identification was clinically acceptable, there was also a significant proportion that did not identify the correct landmarks. These rates are within the range of previously reported failure rates. These data suggest that training and technology that helps identify the correct landmarks are needed over rapidity of the procedure.

\section{Acknowledgements}

The authors would like to thank Dr. Yimage Ahmed and Ms. Nguvan Uhaa.
References

1. Warner KJ, Copass MK, Bulger EM. Paramedic use of needle thoracostomy in the prehospital environment. Prehosp Emerg Care. 2008;12(2):162-168.

2. Schauer SG, Bellamy MA, Mabry RL, Bebarta VS. A comparison of the incidence of cricothyrotomy in the deployed setting to the emergency department at a Level 1 military trauma center: a descriptive analysis. Mil Med. 2015;180(3 Suppl):60-63.

3. Hubble MW, Wilfong DA, Brown LH, Hertelendy A, Benner RW. A meta-analysis of prehospital airway control techniques part II: alternative airway devices and cricothyrotomy success rates. Prehosp Emerg Care. 2010;14(4):515-530.

4. Mabry RL. An analysis of battlefield cricothyrotomy in Iraq and Afghanistan. J Spec Oper Med. 2012;12(1):17-23.
5. Lesperance RN, Carroll CM, Aden JK, Young JB, Nunez TC. Failure rate of prehospital needle decompression for tension pneumothorax in trauma patients. Am Surg. 2018;84(11):1750-1755.

6. Wernick B, Hon HH, Mubang RN, et al. Complications of needle thoracostomy: a comprehensive clinical review. Int J Crit Illn Inj Sci. 2015;5(3):160-169.

7. Weichenthal L, Crane D, Rond L. Needle thoracostomy in the prehospital setting: a retrospective observational study. Prehosp Emerg Care. 2016;20(3):399-403.

8. von Elm E, Altman DG, Egger M, et al. Strengthening the Reporting of Observational Studies in Epidemiology (STROBE) statement: guidelines for reporting observational studies. BMJ. 2007;335(7624):806-808. 